\title{
Apathy and intrinsic functional connectivity networks in amnestic mild cognitive impairment
}

\author{
This article was published in the following Dove Press journal: \\ Neuropsychiatric Disease and Treatment \\ 23 December 2016 \\ Number of times this article has been viewed
}

\author{
Soo Hyun Joo' \\ Chang Uk Lee' \\ Hyun Kook Lim² \\ 'Department of Psychiatry, \\ Seoul St Mary's Hospital, Seoul, \\ ${ }^{2}$ Department of Psychiatry, Saint \\ Vincent Hospital, College of Medicine, \\ The Catholic University of Korea, \\ Suwon, Republic of Korea
}

Background: Although several prior works reported that apathy is associated with conversion to Alzheimer's disease in individuals with amnestic mild cognitive impairment (aMCI), effects of apathy on the functional connectivity (FC) of the brain remain unclear. In this study, we assessed the pattern of association between apathy and default mode network (DMN), salience network and central executive network (CEN) in aMCI subjects.

Methods: Fifty subjects with aMCI and 50 controls (CONs) participated in this study. They underwent clinical assessments and magnetic resonance imaging for the structural and restingstate scan. We explored the patterns of association between apathy inventory (IA) total score and the whole-brain voxel-wise FCs of the DMN, salience network and CEN in aMCI subjects.

Results: We observed that the FCs of the DMN were less and those of CEN were more in the aMCI group than the CON group. Total IA score was negatively correlated with FCs of the anterior cingulate within the DMN, and positively correlated with FCs of the middle frontal, inferior frontal, and supramarginal gyrus within the CEN in the aMCI group.

Conclusion: Our findings suggest that distinctive patterns of association between apathy and FCs in the DMN and CEN in the aMCI group might reflect the putative role of functional network change in the development of apathy in aMCI.

Keywords: apathy, amnestic mild cognitive impairment, intrinsic connectivity networks

\section{Introduction}

Mild cognitive impairment (MCI) and Alzheimer's disease (AD) are accompanied by a number of neuropsychiatric symptoms, as well as cognitive decline. Among the neuropsychiatric symptoms, apathy is the most frequent symptom, independent of AD severity. ${ }^{1}$ Apathy is also prevalent in subjects with $\mathrm{MCI},{ }^{2,3}$ occurring in more than a third of individuals. ${ }^{3}$ Apathy is defined as a syndrome with diminished motivation, initiative and interest, and blunting of emotions. ${ }^{4}$ Several prior works reported that apathy was associated with progression to AD in amnestic MCI (aMCI) individuals, ${ }^{5}$ executive dysfunction and impairment of activities of daily living. ${ }^{6-8}$

To date, previous studies attempted to explore the neural substrates of apathy in AD. Studies using different modalities including structural magnetic resonance imaging (MRI), positron emission tomography (PET) and single photon emission computed tomography (SPECT) have shown associations among apathy and cortical thinning, white matter integrity change, hypometabolism and hypoperfusion in the orbitofrontal, anterior cingulate and inferior temporal areas in the brains of AD patients. ${ }^{9-15}$ Additionally, several studies showed that the presence of white matter lesions was associated with apathy in aMCI patients. ${ }^{16,17}$ Although strong evidence indicates that apathy is correlated with these specific brain damages that might affect disconnection of the cortico-subcortical network, the direct effects of apathy on the functional connectivity $(\mathrm{FC})$ of the brain remain unclear. 
Resting-state functional MRI (RS-fMRI) has recently been highlighted as a new technique for investigating functional intrinsic connectivity networks (ICNs) without an experimentally determined context in patients with dementia. ${ }^{18}$ Functional brain network models are useful to understand the pathophysiology because they consider how neural systems are distributed across the entire brain instead of a specific brain area. ${ }^{19}$ To the best of our knowledge, only one study on subjects with MCI has been performed to investigate the relationship between apathy and ICNs. ${ }^{20}$ This study assessed four different brain networks: the fronto-parietal control network (FPCN; aka central executive network [CEN]), default mode network (DMN), ventral attention network (aka salience network [SN]) and dorsal attention network (DAN), using the neuropsychiatric inventory. The results of the study showed a significant association between increased affective symptoms, in particular, apathy and reduced RS FPCN connectivity. However, they could not show association between apathy and other networks such as the DMN or SN. This might be due to lack of normal distribution of lower apathy score (mean $\pm \mathrm{SD} ; 0.3 \pm 1.0$ ) of the study participants. Moreover, as the study only investigated associations between the mean FC of the resting-state network (RSN) and apathy score, it was hard to find out more sophisticated voxel-wise regional FC changes within the large-scale functional networks (DMN, SN, and FPCN) due to apathy of aMCI subjects.

To overcome the limitations of the previous study, we investigated the distinctive patterns of association between apathy severity and the whole-brain voxel-wise FC of the three large-scale ICNs (DMN, SN and CEN) in aMCI subjects with "clinically significant" apathetic symptom. Based on the literature cited, we conceptualized the apathy in aMCI as the behavioral expression of an abnormal RS FC of the brain and hypothesized that apathetic subjects would exhibit aberrant FC changes in the three ICNs.

\section{Materials and methods}

\section{Study participants}

In this study, 50 subjects with aMCI were included. They were recruited through the outpatient geriatric psychiatry clinic of St Vincent's Hospital from January 2013 to February 2015. All subjects were Korean and right handed. Patients with aMCI met the Petersen's criteria: $\left.{ }^{21} 1\right)$ memory complaint, preferably corroborated by an informant; 2) objective memory impairment for age, education, and gender; 3) essentially preserved general cognitive function; 4) largely intact activities of daily living; and 5) not demented. All aMCI patients had an overall Clinical Dementia Rating ${ }^{22}$ of 0.5 . Objective memory impairment was defined as a performance score of $1.5 \mathrm{SD}$ below the respective age-, education- and gender-specific normative means in at least one of the four episodic memory tests included in the Korean version of Consortium to Establish a Registry for Alzheimer's disease (CERAD-K) neuropsychological battery, namely the word list memory(WLM), word list recall (WLR), word list recognition (WLRc), and constructional recall (CR) tests. ${ }^{23}$

The severity of apathy was assessed using the Apathy Inventory (IA), ${ }^{24}$ that is based on the neuropsychiatric inventory model. ${ }^{25}$ Patients who had a score of 4 or more on the total IA scale with clinically significant apathy were included in the aMCI group..$^{24}$

Study participants who had other neurological or psychiatric conditions were excluded. Fifty healthy control (CON) subjects were recruited within the community through advertisement in a local newspaper. CON subjects were matched to the patients on age, handedness, and level of education.

The study was approved and conducted in accordance with the ethical and safety guidelines set forth by the local Institutional Review Board of the Catholic University of Korea. Written informed consent was obtained from all participants.

\section{MRI acquisition}

Imaging data were collected at the Department of Radiology, St Vincent's Hospital, The Catholic University of Korea, using a 3T Siemens Verio machine and eightchannel Siemens head coil. The parameters of T1-weighted volumetric magnetization-prepared rapid gradient echo scan sequences were TE $=2.5 \mathrm{~ms}$, TR $=1,900 \mathrm{~ms}$, inversion time $=900 \mathrm{~ms}, \mathrm{FOV}=250 \mathrm{~mm}$, matrix $=256 \times 256$, and voxel size $=1.0 \times 1.0 \times 1.0 \mathrm{~mm}^{3}$. RS functional images were collected using a $\mathrm{T} 2 *$ weighting gradient echo sequence with TR $=2,490 \mathrm{~ms}, \mathrm{TE}=30 \mathrm{~ms}$, matrix $=128 \times 128 \times 29$, and voxel size $=2 \times 2 \times 3 \mathrm{~mm}^{3}$. One hundred and fifty volumes were acquired over 5 minutes with the instruction "keep your eyes closed and think of nothing in particular".

\section{Data analysis}

In this study, we used a seed-to-voxel analysis method for evaluation of effect of apathy on FC of three ICNs. We produced individual subject seed-to-voxel connectivity maps using the CONN-fMRI Functional Connectivity toolbox v1.5 (http:// www.nitrc.org/projects/conn). Slice time correction, realignment, normalization, and spatial smoothing were performed for preprocessing of images with a $6 \mathrm{~mm}$ kernel, and the images were temporally band-pass filtered $(0.009<f<0.08 \mathrm{~Hz})$. 
A component-based noise correction method was used to remove physiological and other noise artifacts. ${ }^{26}$ These methods involved extraction of the signals from white matter and cerebrospinal fluid regions with movement-related covariates. The seed region of interests (ROIs) consisting of spheres $6 \mathrm{~mm}$ in radius centered on Montreal Neurological Institute (MNI) coordinates (Montreal, QC, Canada) were used to identify the corresponding networks in prior studies. Each RSN and its corresponding seed ROI (in MNI coordinates) are as follows: DMN (posterior cingulate cortex: 1, -55, 17), CEN (left and right dorsolateral cortex: $-42,34,20 / 44,36,20$ ), and SN (left and right frontoinsular cortex: $-32,26,-14 / 38,22,-10$ ).

For each subject, the average blood oxygen leveldependent time course of each seed was extracted from the functional echo planar images, and then it was correlated with the time courses of whole-brain voxels, using Pearson correlation analysis. Fisher's $r$-to- $z$ transformation of correlation coefficients was conducted for general linear model (GLM) analysis. In the second-level analysis, connectivity maps from all participants were entered into GLM, that was used for measuring the group differences of the FC for each ICN with age, gender, and education as nuisance variables. To examine the relationships between apathy severity and $\mathrm{FC}$ of the ICNs in the aMCI group, the total IA scores were correlated with FCs of the DMN, the SN, and the CEN using the GLM. Statistical significance was thresholded at FDRcorrected $P<0.05$ for multiple-comparison correction.

Table I Demographic and clinical characteristics of the study participants

\begin{tabular}{|c|c|c|c|}
\hline & $\begin{array}{l}\text { CON group } \\
(\mathrm{N}=50)\end{array}$ & $\begin{array}{l}\text { aMCl group } \\
(\mathrm{N}=50)\end{array}$ & $P$-value \\
\hline Age $( \pm S D)$ (years) & $71.2 \pm 4.3$ & $72.1 \pm 3.8$ & NS \\
\hline Education $( \pm S D)$ (years) & $9.4 \pm 3.1$ & $9.0 \pm 4.2$ & NS \\
\hline Gender (M:F) & $22: 28$ & $23: 27$ & NS \\
\hline CDR $( \pm S D)$ & 0 & 0.5 & \\
\hline \multicolumn{4}{|l|}{ CERAD-K battery $( \pm S D)$} \\
\hline VF & $13.3 \pm 3.9$ & $13.2 \pm 3.9$ & NS \\
\hline BNT & $12.7 \pm 2.1$ & $11.8 \pm 2.3$ & NS \\
\hline MMSE & $28.4 \pm 1.5$ & $24.4 \pm 3.3$ & $<0.0001$ \\
\hline WLM & $18.5 \pm 4.5$ & $7.0 \pm 3.4$ & $<0.0001$ \\
\hline $\mathrm{CP}$ & $9.4 \pm 1.5$ & $9.2 \pm 1.9$ & NS \\
\hline WLR & $7.7 \pm 1.8$ & $2.3 \pm 1.8$ & $<0.0001$ \\
\hline WLRc & $9.9 \pm 1.2$ & $3.5 \pm 1.7$ & $<0.0001$ \\
\hline CR & $6.7 \pm 2.9$ & $3.2 \pm 2.9$ & $<0.0001$ \\
\hline IA score (SD) & 0 & $9.3 \pm 2.2$ & $<0.0001$ \\
\hline
\end{tabular}

Abbreviations: aMCl, amnestic mild cognitive impairment; BNT, 15-item Boston Naming Test; CDR, Clinical Dementia Rating; CERAD-K, Korean version of Consortium to Establish a Registry for Alzheimer's Disease; CON, control; CP, constructional praxis; $C R$, constructional recall; $F$, female; IA, apathy inventory; M, male; MMSE, Mini Mental Status Examination; NS, nonsignificant; SD, standard deviation; VF, verbal fluency; WLM, word list memory; WLR, word list recall; WLRc, word list recognition.

\section{Results}

\section{Baseline demographic and clinical data}

Table 1 shows the baseline demographic data of the two subject groups. The Kolmogorov-Smirnov test showed that all variables including total IA scores and CERAD-K neuropsychological test scores were normally distributed. There were no significant differences in gender, age or education between the aMCI and CON groups. Compared with the CON group, the aMCI group showed significantly poorer performances in the Mini Mental Status Examination, WLM, WLR, WLRc, and CR in CERAD-K neuropsychological battery $(P<0.05)$.

\section{Group differences in FC}

Figure 1 shows the statistical map representing the DMN, $\mathrm{SN}$, and CEN determined across all subjects.

Group analysis showed lower FC in the DMN in the aMCI group compared to the CON group in the anterior cingulate cortex and posterior cingulate. In the SN, there were no significant differences between the aMCI and the CON groups in the FCs. The FC in the left middle temporal gyrus and right supramarginal gyrus was significantly higher within the $\mathrm{CEN}$ in the aMCI group compared to the CON group (Table 2 and Figure 2; FDR-corrected $P<0.05$ ).

Figure 2 shows the results of the analysis of correlation between the FCs of the DMN, SN, and CEN and the total IA score in the aMCI group. In the DMN, the FC of the anterior cingulate was negatively correlated with total IA score. In the CEN, the FCs of the middle frontal, inferior frontal, and supramarginal gyrus were positively correlated with the total IA score (Table 2; FDR-corrected $P<0.05$ ). There was no significant difference between the FC of the $\mathrm{SN}$ and the total IA score in the aMCI group.

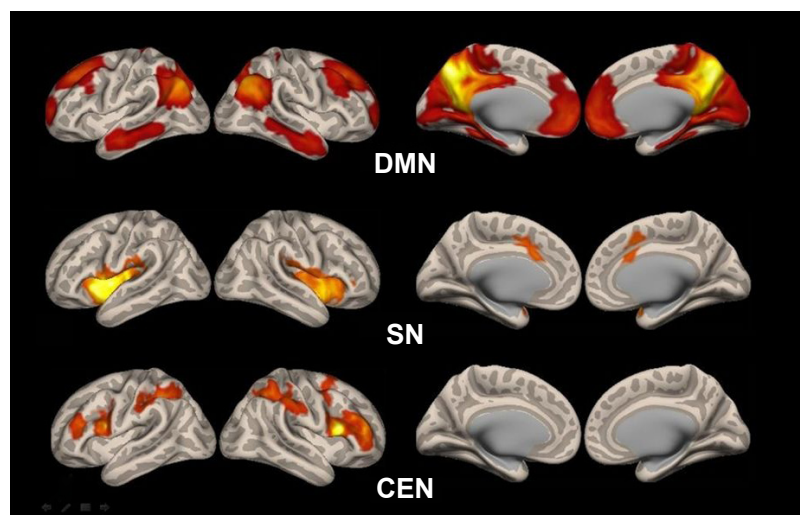

Figure I Statistical map representing the DMN, SN, and CEN determined across all subjects.

Abbreviations: CEN, central executive network; DMN, default mode network; $\mathrm{SN}$, salience network. 
Table 2 Whole-brain voxel-wise FC analysis results

\begin{tabular}{|c|c|c|c|c|c|c|}
\hline $\begin{array}{l}\text { Region } \\
\text { Group differences }\end{array}$ & L/R & Cluster & $P$-value ${ }^{a}$ & \multicolumn{3}{|c|}{ MNI coordinate $(x, y, z)$} \\
\hline \multicolumn{7}{|l|}{ Default mode network } \\
\hline \multicolumn{7}{|l|}{$\mathrm{CON}>\mathrm{aMCl}$} \\
\hline Anterior cingulate & $\mathrm{R}$ & 287 & 0.02 & 2 & 20 & -12 \\
\hline Posterior cingulate & L & 283 & 0.01 & -6 & -42 & 26 \\
\hline Posterior cingulate & $\mathrm{R}$ & 164 & 0.01 & 12 & -38 & 30 \\
\hline \multicolumn{7}{|l|}{ Central executive network } \\
\hline \multicolumn{7}{|l|}{$\mathrm{CON}<\mathrm{aMCl}$} \\
\hline Supramarginal gyrus & $\mathrm{R}$ & 558 & 0.02 & 72 & -42 & -4 \\
\hline Middle temporal gyrus & L & 376 & 0.02 & -52 & -34 & 6 \\
\hline \multicolumn{7}{|l|}{ Total IA score-aMCI FC correlations } \\
\hline \multicolumn{7}{|l|}{ Default mode network } \\
\hline Anterior cingulate & L & 382 & 0.02 & -2 & 36 & 18 \\
\hline \multicolumn{7}{|l|}{ Central executive network } \\
\hline Inferior frontal gyrus pars opercularis & $\mathrm{L}$ & 478 & 0.01 & -52 & 16 & 22 \\
\hline Inferior frontal gyrus pars opercularis & $\mathrm{R}$ & 97 & 0.01 & 50 & 30 & 14 \\
\hline Supramarginal gyrus & $\mathrm{L}$ & 109 & 0.01 & -58 & -22 & 40 \\
\hline Middle frontal gyrus & $\mathrm{R}$ & 109 & 0.03 & 46 & 6 & 32 \\
\hline Frontal pole & $\mathrm{L}$ & 106 & 0.02 & -40 & 36 & 4 \\
\hline
\end{tabular}

Note: ${ }^{\mathrm{F} D D R}$-corrected $\mathrm{P}$-values for the multiple comparisons.

Abbreviations: aMCl, amnestic mild cognitive impairment; CON, control; FC, functional connectivity; FDR, false discovery rate; IA, apathy inventory; L, left; MNI, Montreal Neurological Institute; R, right.

\section{Discussion}

Although this study is not the first RS-fMRI study on the apathy in aMCI, it is the first study to elaborate the relationships between apathy severity and FCs of the large-scale ICNs such as the DMN, the SN, and the CEN.

In this study, we found that the FCs of the DMN were lower and those of the CEN were higher in the aMCI group than in the CON group, that were partially in line with the previous studies. DMN's activity during rest is necessary for memory consolidation. ${ }^{27}$ Additionally, amyloid deposition,

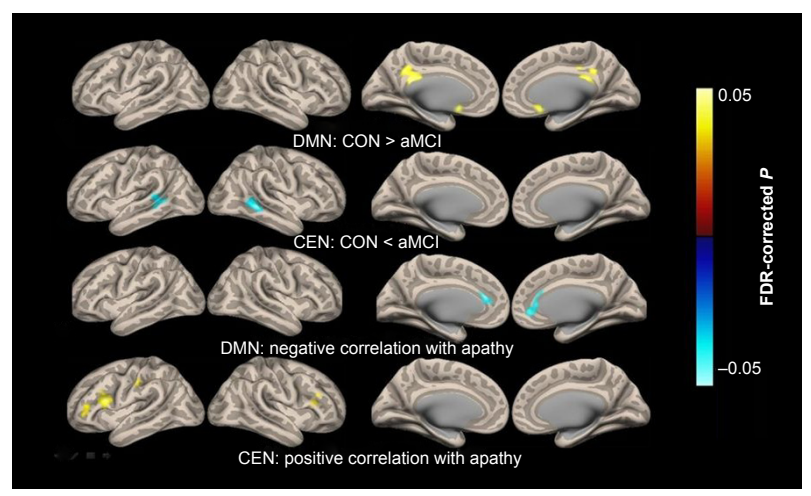

Figure 2 Analysis of correlation between the FCs of the DMN, SN, and CEN and the total IA score in the aMCl group.

Abbreviations: aMCl, amnestic mild cognitive impairment; CEN, central executive network; CON, control; DMN, default mode network; FCs, functional connectivities; FDR, false discovery rate; IA, apathy inventory; SN, salience network. one of the hallmark pathologies of $\mathrm{AD}$, is found initially and most prominently in DMN hubs. ${ }^{28-30}$ For these reasons, the $\mathrm{DMN}$ is the most frequently studied network in dementia. Loss of FC in the DMN is a well-established finding in AD patients, but it is inconsistent in MCI patients. Our findings showing a decreased FC in the DMN of MCI patients are consistent with previous studies. ${ }^{31-34}$ However, the changes of the DMN in MCI patients remain controversial. A previous study showed the selective disruption of the DMN in MCI patients, indicating both decreased and increased DMN connectivity. ${ }^{11}$ Moreover, the most recent study on MCI patients presented increased DMN connectivity. ${ }^{35}$ Gardini et al explained that increased FC of the DMN in aMCI subjects may reflect a maladaptive mechanism that would represent the damaged brain's attempt to cope with regional atrophy or amyloid deposition in these areas. ${ }^{36}$ Based on the current uncertainty inherent in the clinical concept of $\mathrm{MCI},{ }^{37,38}$ controversial changes of DMN in aMCI patients may be due to the heterogeneity of the aMCI population between the studies.

Besides the DMN, SN and CEN play important roles in cognitive processing. ${ }^{39}$ The SN plays a crucial role in switching between the CEN and the DMN. ${ }^{40}$ Once a salient event is detected, the SN initiates the appropriate transient control signals to engage the CEN to mediate attention, working memory, and executive control, all while disengaging the 
DMN. ${ }^{41}$ Negative correlations (anticorrelations) between the DMN and the CEN or SN support the reciprocal relationship between these ICNs. ${ }^{41,42}$ Other RS-fMRI studies explained that the greater FCs of the CEN and SN might be due to a compensatory mechanism associated with DMN impairments. ${ }^{31,43}$ Therefore, the results that showed decreased FCs of the DMN and increased FCs of the CEN in the aMCI group could be interpreted by the DMN impairment associated with disease progression and aberrant hyperactivity of CEN associated with a compensatory process.

We also found that total IA score of the aMCI group was negatively correlated with the $\mathrm{FC}$ of the anterior cingulate within the DMN and positively correlated with the FC of the middle frontal, inferior frontal, and supramarginal gyrus within the CEN in the aMCI group. Apathy is a complex syndrome with lack of initiation, lack of interest, and reduced emotional responsiveness ${ }^{4}$ due to direct or indirect involvement of the prefrontal lobes. ${ }^{44}$ Neural substrates associated with apathy were found in several studies in AD spectrum disorders. An SPECT imaging study found that apathy was negatively correlated with perfusion in the right anterior cingulate cortex, right middle orbitofrontal gyrus, and left superior dorsolateral prefrontal cortex. ${ }^{9}$ An MRI study using voxel-based morphometry found that apathy was associated with atrophy of the anterior cingulate, ventrolateral orbitofrontal cortex, and left insula in AD.${ }^{45}$ Other studies using MRI with diffusion tensor imaging have shown relationships between increased white matter abnormalities and apathy in MCI and AD. ${ }^{15,17,46}$ A recent study found that the presence of lesions in the anterior thalamic radiations was associated with the severity of apathy in aMCI. ${ }^{16}$ These studies confirmed the evidence of white matter disconnection associated with apathy and support the view that alterations in the neural mechanisms of apathy occur in the early course of AD. Furthermore, a recent RS-fMRI study revealed that a decreased FPCN was associated with greater apathy in MCI. ${ }^{20}$ Our findings build upon previous work by suggesting FC changes in the three ICNs for individuals with aMCI who have greater apathy. Also, our result of association between apathy and anterior cingulate coincided with many existing reports in $\mathrm{AD}^{3,46-48}$ The anterior cingulate is one of the important anatomical structures involved in motivation. The mesocortico-limbic pathway is a dopaminergic network from the ventral tegmentum ${ }^{49}$ to the different anterior cortical regions (frontal and parietal) connecting via the anterior cingulate. ${ }^{50}$ Therefore, the disruption of connections between the anterior cingulate and other cortical and subcortical regions would lead to the mechanism of apathy. ${ }^{51}$
The present study has several limitations. First, all presented data are cross-sectional in nature. Further longitudinal studies will be needed to clarify the large-scale functional network changes underlying apathy in the AD progression. Second, as we recruited ICNs that were most similar to the previously identified networks template, other networks of interest such as the DAN could not be observed. Third, we did not account for use of psychotropic medications such as antidepressants, cholinesterase inhibitors, dopamine agonists or stimulants such as methylpenidate or modafinil that reportedly help patients with apathy. Despite these limitations, this study showed that apathy is associated with an alteration of large-scale functional brain networks, that included not only the intra-networks but also inter-networks of the DMN, SN, and CEN. Additionally, we included a relatively large number of subjects compared with the studies previously reported.

\section{Conclusion}

We showed altered FCs of the DMN and CEN and distinctive patterns of correlations between apathy and FCs in the DMN and CEN in the aMCI group. These results might reflect putative role of functional network change in the development of apathy in aMCI. Additional large and longitudinal studies will help further elucidate the underlying neurobiological mechanism of apathy in AD progression.

\section{Acknowledgment}

This research was supported by Basic Science Research Program through the National Research Foundation of Korea funded by the Ministry of Science, ICT, and Future Planning (NRF-2015R1C1A1A02036578).

\section{Disclosure}

The authors report no conflicts of interest in this work.

\section{References}

1. Robert PH, Verhey FR, Byrne EJ, et al. Grouping for behavioral and psychological symptoms in dementia: clinical and biological aspects. Consensus paper of the European Alzheimer disease consortium. Eur Psychiatry. 2005;20(7):490-496.

2. Geda YE, Roberts RO, Knopman DS, et al. Prevalence of neuropsychiatric symptoms in mild cognitive impairment and normal cognitive aging: population-based study. Arch Gen Psychiatry. 2008;65(10): 1193-1198.

3. Apostolova LG, Cummings JL. Neuropsychiatric manifestations in mild cognitive impairment: a systematic review of the literature. Dement Geriatr Cogn Disord. 2008;25(2):115-126.

4. Starkstein SE, Petracca G, Chemerinski E, Kremer J. Syndromic validity of apathy in Alzheimer's disease. Am J Psychiatry. 2001;158(6): 872-877.

5. Palmer K, Di Iulio F, Varsi AE, et al. Neuropsychiatric predictors of progression from amnestic-mild cognitive impairment to Alzheimer's disease: the role of depression and apathy. J Alzheimers Dis. 2010;20(1): $175-183$. 
6. Boyle PA, Malloy PF, Salloway S, Cahn-Weiner DA, Cohen R, Cummings JL. Executive dysfunction and apathy predict functional impairment in Alzheimer disease. Am J Geriatr Psychiatry. 2003;11(2): 214-221.

7. McPherson S, Fairbanks L, Tiken S, Cummings JL, Back-Madruga C. Apathy and executive function in Alzheimer's disease. J Int Neuropsychol Soc. 2002;8(3):373-381.

8. Marshall GA, Rentz DM, Frey MT, Locascio JJ, Johnson KA, Sperling RA. Executive function and instrumental activities of daily living in mild cognitive impairment and Alzheimer's disease. Alzheimers Dement. 2011;7(3):300-308.

9. Benoit M, Clairet S, Koulibaly PM, Darcourt J, Robert PH. Brain perfusion correlates of the apathy inventory dimensions of Alzheimer's disease. Int J Geriatr Psychiatry. 2004;19(9):864-869.

10. Rosen HJ, Allison SC, Schauer GF, Gorno-Tempini ML, Weiner MW, Miller BL. Neuroanatomical correlates of behavioural disorders in dementia. Brain. 2005;128(Pt 11):2612-2625.

11. Robert PH, Darcourt G, Koulibaly MP, et al. Lack of initiative and interest in Alzheimer's disease: a single photon emission computed tomography study. Eur J Neurol. 2006;13(7):729-735.

12. Marshall GA, Monserratt L, Harwood D, Mandelkern M, Cummings JL, Sultzer DL. Positron emission tomography metabolic correlates of apathy in Alzheimer disease. Arch Neurol. 2007;64(7):1015-1020.

13. Guercio BJ, Donovan NJ, Ward A, et al. Apathy is associated with lower inferior temporal cortical thickness in mild cognitive impairment and normal elderly individuals. $J$ Neuropsychiatry Clin Neurosci. 2015;27(1):e22-e27.

14. Donovan NJ, Wadsworth LP, Lorius N, et al. Regional cortical thinning predicts worsening apathy and hallucinations across the Alzheimer disease spectrum. Am J Geriatr Psychiatry. 2014;22(11):1168-1179.

15. Hahn C, Lim HK, Won WY, Ahn KJ, Jung WS, Lee CU. Apathy and white matter integrity in Alzheimer's disease: a whole brain analysis with tract-based spatial statistics. PLoS One. 2013;8(1):e53493.

16. Torso M, Serra L, Giulietti G, et al. Strategic lesions in the anterior thalamic radiation and apathy in early Alzheimer's disease. PLoS One. 2015;10(5): 0124998

17. Cacciari C, Moraschi M, Di Paola M, et al. White matter microstructure and apathy level in amnestic mild cognitive impairment. $J$ Alzheimers Dis. 2010;20(2):501-507.

18. Vemuri P, Jones DT, Jack CR Jr. Resting state functional MRI in Alzheimer's Disease. Alzheimers Res Ther. 2012;4(1):2.

19. Yuen GS, Gunning-Dixon FM, Hoptman MJ, et al. The salience network in the apathy of late-life depression. Int J Geriatr Psychiatry. 2014; 29(11):1116-1124.

20. Munro CE, Donovan NJ, Guercio BJ, et al. Neuropsychiatric symptoms and functional connectivity in mild cognitive impairment. $J$ Alzheimers Dis. 2015;46(3):727-735.

21. Petersen RC. Mild cognitive impairment as a diagnostic entity. J Intern Med. 2004;256(3):183-194.

22. Morris JC. The Clinical Dementia Rating (CDR): current version and scoring rules. Neurology. 1993;43(11):2412-2414.

23. Lee JH, Lee KU, Lee DY, et al. Development of the Korean version of the Consortium to Establish a Registry for Alzheimer's Disease Assessment Packet (CERAD-K): clinical and neuropsychological assessment batteries. J Gerontol B Psychol Sci Soc Sci. 2002;57(1):P47-P53.

24. Robert PH, Clairet S, Benoit M, et al. The apathy inventory: assessment of apathy and awareness in Alzheimer's disease, Parkinson's disease and mild cognitive impairment. Int J Geriatr Psychiatry. 2002; 17(12):1099-1105.

25. Cummings JL. The Neuropsychiatric Inventory: assessing psychopathology in dementia patients. Neurology. 1997;48(5 Suppl 6): S10-S16.

26. Behzadi Y, Restom K, Liau J, Liu TT. A component based noise correction method (CompCor) for BOLD and perfusion based fMRI. Neuroimage. 2007;37(1):90-101.
27. Fox MD, Raichle ME. Spontaneous fluctuations in brain activity observed with functional magnetic resonance imaging. Nat Rev Neurosci. 2007;8(9):700-711.

28. Buckner RL, Andrews-Hanna JR, Schacter DL. The brain's default network: anatomy, function, and relevance to disease. Ann N Y Acad Sci. 2008;1124:1-38.

29. Hedden T, Van Dijk KR, Becker JA, et al. Disruption of functional connectivity in clinically normal older adults harboring amyloid burden. J Neurosci. 2009;29(40):12686-12694.

30. Mormino EC, Smiljic A, Hayenga AO, et al. Relationships between betaamyloid and functional connectivity in different components of the default mode network in aging. Cereb Cortex. 2011;21(10):2399-2407.

31. Agosta F, Pievani M, Geroldi C, Copetti M, Frisoni GB, Filippi M. Resting state fMRI in Alzheimer's disease: beyond the default mode network. Neurobiol Aging. 2012;33(8):1564-1578.

32. Bai F, Zhang Z, Yu H, et al. Default-mode network activity distinguishes amnestic type mild cognitive impairment from healthy aging: a combined structural and resting-state functional MRI study. Neurosci Lett. 2008;438(1):111-115.

33. Sorg C, Riedl V, Muhlau M, et al. Selective changes of resting-state networks in individuals at risk for Alzheimer's disease. Proc Natl Acad Sci U S A. 2007;104(47):18760-18765.

34. Wang Y, Risacher SL, West JD, et al. Altered default mode network connectivity in older adults with cognitive complaints and amnestic mild cognitive impairment. J Alzheimers Dis. 2013;35(4):751-760.

35. Qi Z, Wu X, Wang Z, et al. Impairment and compensation coexist in amnestic MCI default mode network. Neuroimage. 2010;50(1):48-55.

36. Gardini S, Venneri A, Sambataro F, et al. Increased functional connectivity in the default mode network in mild cognitive impairment: a maladaptive compensatory mechanism associated with poor semantic memory performance. J Alzheimers Dis. 2015;45(2):457-470.

37. Sinai M, Phillips NA, Chertkow H, Kabani NJ. Task switching performance reveals heterogeneity amongst patients with mild cognitive impairment. Neuropsychology. 2010;24(6):757-774.

38. Delano-Wood L, Bondi MW, Sacco J, et al. Heterogeneity in mild cognitive impairment: differences in neuropsychological profile and associated white matter lesion pathology. J Int Neuropsychol Soc. 2009; 15(6):906-914

39. Menon V. Large-scale brain networks and psychopathology: a unifying triple network model. Trends Cogn Sci. 2011;15(10):483-506.

40. Sridharan D, Levitin DJ, Menon V. A critical role for the right frontoinsular cortex in switching between central-executive and default-mode networks. Proc Natl Acad Sci U S A. 2008;105(34):12569-12574.

41. Menon V, Uddin LQ. Saliency, switching, attention and control: a network model of insula function. Brain Struct Funct. 2010; 214(5-6):655-667.

42. Uddin LQ, Kelly AM, Biswal BB, Castellanos FX, Milham MP. Functional connectivity of default mode network components: correlation, anticorrelation, and causality. Hum Brain Mapp. 2009;30(2):625-637.

43. Machulda MM, Jones DT, Vemuri P, et al. Effect of APOE epsilon4 status on intrinsic network connectivity in cognitively normal elderly subjects. Arch Neurol. 2011;68(9):1131-1136.

44. Ghika-Schmid F, Bogousslavsky J. The acute behavioral syndrome of anterior thalamic infarction: a prospective study of 12 cases. Ann Neurol. 2000;48(2):220-227.

45. Stanton BR, Leigh PN, Howard RJ, Barker GJ, Brown RG. Behavioural and emotional symptoms of apathy are associated with distinct patterns of brain atrophy in neurodegenerative disorders. J Neurol. 2013; 260(10):2481-2490.

46. Kim JW, Lee DY, Choo IH, et al. Microstructural alteration of the anterior cingulum is associated with apathy in Alzheimer disease. Am J Geriatr Psychiatry. 2011;19(7):644-653.

47. Bruen PD, McGeown WJ, Shanks MF, Venneri A. Neuroanatomical correlates of neuropsychiatric symptoms in Alzheimer's disease. Brain. 2008;131(Pt 9):2455-2463. 
48. Ota M, Sato N, Nakata Y, Arima K, Uno M. Relationship between apathy and diffusion tensor imaging metrics of the brain in Alzheimer's disease. Int J Geriatr Psychiatry. 2012;27(7):722-726.

49. Starkstein SE, Leentjens AF. The nosological position of apathy in clinical practice. J Neurol Neurosurg Psychiatry. 2008;79(10):1088-1092.

50. Marin RS, Fogel BS, Hawkins J, Duffy J, Krupp B. Apathy: a treatable syndrome. J Neuropsychiatry Clin Neurosci. 1995;7(1):23-30.
51. Migneco O, Benoit M, Koulibaly PM, et al. Perfusion brain SPECT and statistical parametric mapping analysis indicate that apathy is a cingulate syndrome: a study in Alzheimer's disease and nondemented patients. Neuroimage. 2001;13(5):896-902.

\section{Publish your work in this journal}

Neuropsychiatric Disease and Treatment is an international, peerreviewed journal of clinical therapeutics and pharmacology focusing on concise rapid reporting of clinical or pre-clinical studies on a range of neuropsychiatric and neurological disorders. This journal is indexed on PubMed Central, the 'PsycINFO' database and CAS, and is the official journal of The International Neuropsychiatric Association (INA). The manuscript management system is completely online and includes a very quick and fair peer-review system, which is all easy to use. Visit http://www.dovepress.com/testimonials.php to read real quotes from published authors.

Submit your manuscript here: http://www.dovepress.com/neuropsychiatric-disease-and-treatment-journal 